\title{
New Bounds for Hypercube Slicing Numbers
}

\author{
M. Reza Emamy-Khansary ${ }^{1}$ and Martin Ziegler $^{2 \dagger}$ \\ ${ }^{1}$ PO Box 23355, Dept. of Math.,University of Puerto Rico,San Juan PR, 00931 \\ ${ }^{2}$ Heinz Nixdorf Institute, University of Paderborn, 33095 Germany
}

received February 4, 2001, revised April 10, 2001, accepted April 16, 2001.

\begin{abstract}
What is the maximum number of edges of the $d$-dimensional hypercube, denoted by $S(d, k)$, that can be sliced by $k$ hyperplanes? This question on combinatorial properties of Euclidean geometry arising from linear separability considerations in the theory of Perceptrons has become an issue on its own. We use computational and combinatorial methods to obtain new bounds for $S(d, k), d \leq 8$. These strengthen earlier results on hypercube cut numbers.
\end{abstract}

Keywords: Hypercube cut number, linear separability, combinatorial geometry

\section{Introduction}

Hyperplane $H \subset \mathbb{R}^{d}$ is said to slice the line segment $L=\{\lambda a+(1-\lambda) b: 0 \leq \lambda \leq 1\}$ between vertices $a, b \in \mathbb{R}^{d}$ iff their intersection $H \cap L$ is an interior point of this segment. Let $\mathcal{G}_{d}=\left(\mathcal{V}_{d}, \mathcal{E}_{d}\right)$ denote the $d$ dimensional hypercube, i.e., the geometric graph on vertex set $\mathcal{V}_{d}=\{-1,+1\}^{d} \subseteq \mathbb{R}^{d}$ with $\left|\mathcal{E}_{d}\right|=d \cdot 2^{d-1}$ undirected edges. It is obvious that the $d$ hyperplanes

$$
H_{j}=\left\{x \in \mathbb{R}^{d}: x_{j}=0\right\} \quad \text { for } j=1 \ldots d
$$

slice all edges of $\mathcal{G}_{d}$. However M. Paterson [13] observed that for $d=6$ one can do better: 5

sophisticatedly chosen hyperplanes suffice to cut all members of $\mathcal{E}_{6}$.

So what is the minimum number $C(d)$ of hyperplanes needed to cut all the edges in $\mathcal{E}_{d}$ for arbitrary $d$ ?

Problems of this kind arise from linear separability questions in connection with Perceptrons [10]. On the other hand, cutting the hypercube has a vast variety of applications in theory of integer linear programming [1], and in optimization of pseudo-

\begin{tabular}{rlr|}
$C(d-1)$ & $\leq C(d) \leq d$ & \\
$C\left(d_{1}+d_{2}\right)$ & $\leq C\left(d_{1}\right)+C\left(d_{2}\right)$ & {$[14]$} \\
$C(4)$ & $>3$ & {$[2]$} \\
$C(5)$ & $>4$ & {$[15]$} \\
$C(6)$ & $\leq 5$ & {$[13]$} \\
$C(d)$ & $\geq \Omega(\sqrt{d})$ & {$[11]$} \\
\hline
\end{tabular}

${ }^{\dagger}$ Partially supported by DFG Grant Me872/7-3 
boolean functions [8]. Notice for instance that $C(d)$ is a lower bound on the size of any threshold circuit [7] computing the parity of $d$ bits, the best known upper bound being linear $O(d)$.

The values of these Cut Numbers $C(d)$ as well as their asymptotic growth have first been raised by O'Neil [11], then by Grünbaum [6], and most recently by Klee [9]. Previous results (see Table 1.1) established $C(d)=d$ for $d=1 \ldots 5$ and $C(6)=5$. But for higher dimensions, only lower and upper bounds are known which leave a quadratic gap as depicted in Figure 2.1.

$$
\Omega(\sqrt{d}) \sim \frac{d \cdot 2^{d-1}}{\lceil d / 2\rceil \cdot(\underset{\lceil d / 2\rceil}{d})} \leq C(d) \leq\left\lceil\frac{5}{6} d\right\rceil \sim O(d)
$$

\section{Motivation}

Notice that for each $d$, exactly one inequality in $C(d) \leq C(d+1) \leq C(d)+1$ is tight, and $C(d)=$ $C(d+1)$ holds for at least every 6th $d$. Improved results about this frequency immediately yield better bounds. We therefore believe that in particular the first occurrence of these 'anomalities' $C(d)=$ $C(d+1)$ deserves further investigation.

Our aim is to study cuts of the 5 and 6 -cubes according to the number of edges they slice. More precisely, we want to determine the Slicing Number $S(d, k)$ : the maximum number of edges of

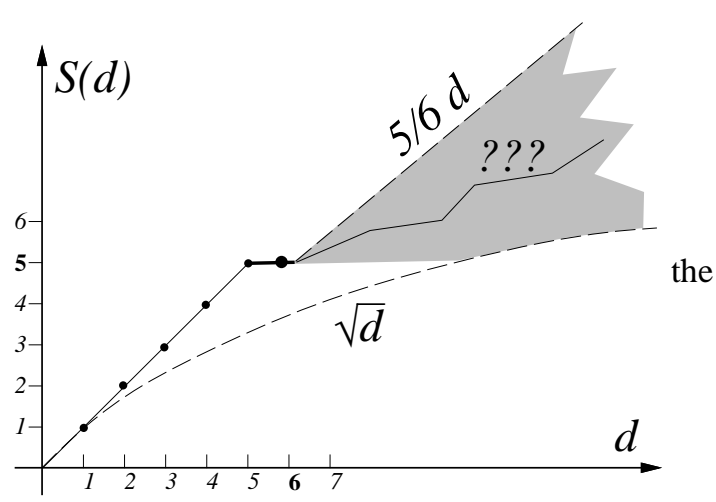

2.1 Figure: Bounds ON CUT Numbers. $d$-cube that $k$ hyperplanes can cut. Obviously, $C(d)>k$ iff $S(d, k)<d \cdot 2^{d-1}$. Importance of these values $S(d, k)$ to the cut number problem was first observed in [3], where the author strengthened his former result [2] from " $C(4)>3$ " to " $S(4,3) \stackrel{!}{=} 30<32=\left|\mathcal{E}_{4}\right|$ ". We recall some more facts regarding $S(d, k)$ :

\subsection{Lemma: Known Properties of Slicing Numbers.}

a) $S(d, k) \leq d \cdot 2^{d-1}$ with equality iff $C(d) \leq k$.

b) $S(d, k) \geq k \cdot 2^{d-1}$ for $k \leq d$, attained by the cuts in (1).

c) $S(d, k)+S(d, l) \geq S(d, k+l) \geq S(d, k)$; in particular $S(d, k) \leq k \cdot S(d, 1)$.

d) For $k=1$, the values are well known [11]: $S(d, 1)=\lceil d / 2\rceil \cdot\left(\begin{array}{c}d \\ \lceil d / 2\rceil\end{array}\right)$

The lower bound in (2) emerges from

$$
d \cdot 2^{d-1} \stackrel{a)}{=} S(d, C(d)) \stackrel{c)}{\leq} \quad C(d) \cdot S(d, 1) \stackrel{d)}{=} \quad C(d) \cdot\lceil d / 2\rceil \cdot\left(\begin{array}{c}
d \\
\lceil d / 2\rceil
\end{array}\right)
$$

Its weakness is at hand: Relation (3) presumes the $k$ different cuts to slice disjoint subsets of edges. This worst case does occur for example in the cuts given by (1). But starting with $d=6$, these cuts are not optimal; and Paterson's optimal 5 cuts (of cardinalities $48+52+52+60+60=272$ ) through the 192 edges of the 6-cube obviously have a large number of overlaps.

Hence the bound in (3) gives a far over-estimate, and better knowledge about $S(d, k)$ immediately yields improved bounds for $C(d)$. 


\section{Results}

In [15], the authors developed computational methods for determining the cut number of the 5-cube. This provided a means to prove $4 \stackrel{!}{<} C(5) \leq C(6) \leq C(7) \leq C(8), \quad$ which implies

$$
S(5,4)<\left|\mathcal{E}_{5}\right|=80, \quad S(6,4)<\left|\mathcal{E}_{6}\right|=192, \quad S(7,4)<\left|\mathcal{E}_{7}\right|=448, \quad \text { and } \quad S(8,4)<\left|\mathcal{E}_{8}\right|=1024 .
$$

In the present work, we strengthen these results by showing

$$
S(5,4)=78, \quad 181 \leq S(6,4) \leq 187, \quad 410 \leq S(7,4) \leq 436, \quad \text { and } \quad 908 \leq S(8,4) \leq 996 .
$$

To this end, we enhanced the algorithms from [15] using techniques from [12]. The computational approach furthermore allows us to determine the exact values of all slicing numbers in dimension 5 (about which hardly anything was known before) as well as $S(6,3)$ and $S(7,2)$. Our algorithms have an overall time-complexity of $2^{O\left(k \cdot d^{2}\right)}$, thus posing hard limits on the tractability of higher dimensions and more cuts. However in connection with the following lemma, the computational results yield lower and upper bounds also for such cases.

\subsection{Lemma: New Properties of Slicing Numbers}

$$
\begin{aligned}
& \text { e) } S(d, k) \geq \sum_{i=0}^{k-1}\left\lceil\frac{d-i}{2}\right\rceil \cdot\left(\begin{array}{c}
d \\
\left\lceil\frac{d-i}{2}\right\rceil
\end{array}\right) \text { for } k \leq d . \\
& \text { f) } S(d, k) \leq\lfloor S(d-1, k) \cdot 2 d /(d-1)\rfloor \\
& \text { g) } S(n+m, k+l) \geq S(n, k) \cdot 2^{m}+S(m, l) \cdot 2^{n}, \quad \text { and thus by induction } \\
& \text { h) } S\left(\sum d_{i}, \sum k_{i}\right) \geq\left(\prod 2^{d_{i}}\right) \cdot \sum S\left(d_{i}, k_{i}\right) / 2^{d_{i}}, \quad S(n d, n k) \geq n 2^{(n-1) d} \cdot S(d, k)
\end{aligned}
$$

Proof: The following hyperplanes cut between neighbouring levels of the cube, that is, sets of vertices with the same number of coordinates equal to 1 :

$$
H_{j}=\left\{x \in \mathbb{R}^{d}: \sum_{i} x_{i}=j\right\}, \quad j=\left\{\begin{aligned}
+1,-1, \pm 3, \ldots, \pm(d-3), \pm(d-1) & : d \text { even } \\
0,+2,-2, \pm 4, \ldots, \pm(d-3), \pm(d-1) & : d \text { odd }
\end{aligned}\right.
$$

It is easy to see that the $i$-th cut slices exactly $\left\lceil\frac{d-i}{2}\right\rceil \cdot\left(\begin{array}{c}d \\ \left\lceil\frac{d-i}{2}\right\rceil\end{array}\right)$ many edges, and different cuts slice disjoint sets of edges.

To show f), consider $k$ cuts in the $d$-cube which slice the maximum number $S(d, k)$ of edges. From these sliced edges, let $m_{i}$ count those which belong to the $i$-th facet, i.e., the $(d-1)$-dimensional subcube for $i=1 \ldots 2 d$. As any edge is contained in exactly $d-1$ such facets, we have

$$
(d-1) \cdot S(d, k)=\sum_{i=1}^{2 d} m_{i} \leq \sum_{i=1}^{2 d} S(d-1, k)=2 d \cdot S(d-1, k) .
$$


For g) consider the $(n+m)$-cube and, for each $\left(x_{1}, \ldots, x_{m}\right) \in \mathcal{V}_{m}$, the subcube $\left(x_{1}, \ldots, x_{m}\right) \times \mathcal{V}_{n}$. Extend the $k$ hyperplanes for the $n$-cube to these $2^{m} \quad n$-dimensional parallel subcubes according to the figure to the right. That way, we slice $S(n, k) \cdot 2^{m}$ edges. Each such edge belongs to some fixed subcube $\left(x_{1}, \ldots, x_{m}\right) \times \mathcal{V}_{n}$ and consequently runs in direction (along dimension) $i$ where $m+1 \leq i \leq$ $m+n$.

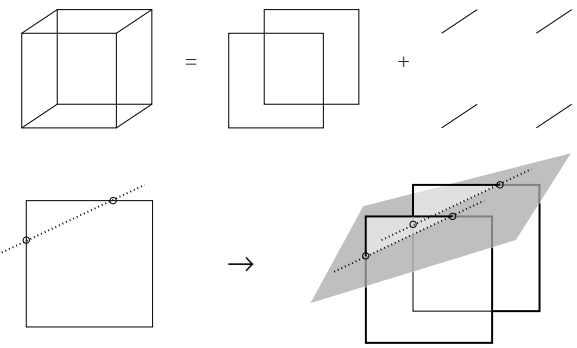

Similarly, the $l$ cuts in the $m$-cube can be extended to slice $S(m, l) \cdot 2^{n}$ edges of the $(n+m)$-cube; these edges run in directions $1, \ldots, m$. The sets of edges sliced by the first $k$ and the second $l$ extended cuts, respectively, are obviously disjoint: they differ in the directions they run.

Property g) strengthens well-known subadditivity of the cut number function [14]. Indeed, ' $C\left(d_{1}+d_{2}\right) \leq$ $C\left(d_{1}\right)+C\left(d_{2}\right)^{\prime}$ is now re-obtained as immediate consequence of $\mathrm{g}$ ) and a): Set $n:=d_{1}, m:=d_{2}, k:=C(n)$, $l:=C(m)$ and observe that

$$
(n+m) \cdot 2^{n+m-1} \stackrel{a)}{\geq} S(n+m, k+l) \stackrel{g}{\geq} S(n, k) \cdot 2^{m}+S(m, l) \cdot 2^{n} \stackrel{a)}{=} n \cdot 2^{n-1} \cdot 2^{m}+m \cdot 2^{m-1} \cdot 2^{n}
$$

Since both ends coincide, we have equality $S(n+m, k+l)=(n+m) \cdot 2^{n+m-1}$ and thus $C\left(d_{1}\right)+C\left(d_{2}\right) \stackrel{\text { def }}{=}$ $k+l \stackrel{a)}{\geq} C(n+m) \stackrel{\text { def }}{=} C\left(d_{1}+d_{2}\right)$. Let us point out that this also shows that the estimate g) cannot be improved in general; neither can $\mathrm{f}$ ) as seen from inserting $k:=C(d+1)$.

Similarly Property h) is stronger than (2) because it implies the latter:

$$
6 n \cdot 2^{6 n-1} \stackrel{a)}{\geq} S(6 n, 5 n) \stackrel{h)}{\geq} n \cdot 2^{6(n-1)} \cdot S(6,5)=6 n \cdot 2^{6 n-1} \stackrel{a)}{\Rightarrow} C(6 n) \leq 5 n .
$$

We now proceed to state the main result of this work:

\subsection{Theorem: Main Result}

\begin{tabular}{|c|c|c|c|c|c|c|c|}
\hline$S(d, k)$ & $k=1$ & 2 & 3 & 4 & 5 & 6 & 7 \\
\hline$d=3$ & 6 & 10 & 12 & 12 & 12 & 12 & 12 \\
\hline$d=4$ & 12 & 24 & 30 & 32 & 32 & 32 & 32 \\
\hline$d=5$ & 30 & 54 & 70 & 78 & 80 & 80 & 80 \\
\hline$d=6$ & 60 & 120 & 160 & $\begin{array}{l}\geq 181 \\
\leq 187\end{array}$ & 192 & 192 & 192 \\
\hline$d=7$ & 140 & 260 & $\begin{array}{l}\geq \mathbf{3 5 0} \\
\leq \mathbf{3 7 3}\end{array}$ & $\begin{array}{l}\geq 410 \\
\leq 436\end{array}$ & $\begin{array}{l}\geq \mathbf{4 3 4} \\
\leq 448\end{array}$ & 448 & 448 \\
\hline$d=8$ & 280 & 560 & $\begin{array}{l}\geq 770 \\
\leq \mathbf{8 5 2}\end{array}$ & $\begin{array}{l}\geq 908 \\
\leq 996\end{array}$ & $\begin{array}{r}\geq \mathbf{9 8 0} \\
\leq 1024\end{array}$ & $\begin{array}{l}\geq \mathbf{1 0 0 8} \\
\leq 1024\end{array}$ & 1024 \\
\hline
\end{tabular}

The following bounds hold for $S(d, k), d \leq 8$. Bold face entries are new.

3.3 TABLE: BOUNDS ON SLICING NUMBERS UP TO DIMENSION 8. 
Proof: The entries for $d \leq 4$ have been known before [3]; also we have those for $k=1$ by d); and $S(d, k)=d \cdot 2^{d-1}$ for $k \geq\left\lceil\frac{5}{6} d\right\rceil \geq C(d)$ by a) and (2). For $k=2$ and even $d$,

$$
2 \cdot S(d, 1) \stackrel{d)}{=}\left\lceil\frac{d}{2}\right\rceil \cdot\left(\begin{array}{c}
d \\
\left\lceil\frac{d}{2}\right\rceil
\end{array}\right)+\left\lceil\frac{d-1}{2}\right\rceil \cdot\left(\begin{array}{c}
d \\
\left\lceil\frac{d-1}{2}\right\rceil
\end{array}\right) \stackrel{e)}{\leq} S(d, 2) \stackrel{c)}{\leq} 2 \cdot S(d, 1)
$$

Cuts listed in Table 3.4 and Table 3.6 slice 181 and 908 edges of $\{-1,+1\}^{6}$ and $\{-1,+1\}^{8}$, respectively. This can be verified by hand or, more conveniently, on our interactive web page [16]. Property e) implies $S(7,5) \geq 434$ and $S(8,6) \geq 1008$. The remaining lower bounds on $S(d, k)$ are attained by taking Cut $d .1$ through Cut $d . k$ from Table 3.7 .

Some upper bounds can be deduced from lower dimensional cases using Property f), see Table 3.5. The remaining upper bounds for $S(5,2), S(5,3), S(5,4), S(6,3)$, and $S(7,2)$ were obtained by computer exhaustive search as described in Section 4.

$$
\begin{aligned}
& 0=-1+2 \cdot x_{1}+2 \cdot x_{2}+2 \cdot x_{3}-4 \cdot x_{4}+2 \cdot x_{5}+2 \cdot x_{6} \\
& 0=1+2 \cdot x_{1}+2 \cdot x_{2}+2 \cdot x_{3}+4 \cdot x_{4}+2 \cdot x_{5}-2 \cdot x_{6} \\
& 0=-4+5 \cdot x_{1}+5 \cdot x_{2}+5 \cdot x_{3}+6 \cdot x_{4}+6 \cdot x_{5}+10 \cdot x_{6} \\
& 0=2+4 \cdot x_{1}+4 \cdot x_{2}+4 \cdot x_{3}-5 \cdot x_{4}+3 \cdot x_{5}-7 \cdot x_{6}
\end{aligned}
$$

$$
\begin{aligned}
& S(6,4) \leq\lfloor S(5,4) \cdot 12 / 5\rfloor=187 \\
& S(7,3) \leq\lfloor S(6,3) \cdot 14 / 6\rfloor=373 \\
& S(7,4) \leq\lfloor S(6,4) \cdot 14 / 6\rfloor \leq 436 \\
& S(8,3) \leq\lfloor S(7,3) \cdot 16 / 7\rfloor \leq 852 \\
& S(8,4) \leq\lfloor S(7,4) \cdot 16 / 7\rfloor \leq 996
\end{aligned}
$$

3.5 TABLE: SOME UPPER BOUNDS.

$$
\begin{aligned}
& 0=-1+2 x_{1}+2 x_{2}+2 x_{3}+2 x_{4}+2 x_{5}-4 x_{6}+2 x_{7}+2 x_{8} \\
& 0=1+2 x_{1}+2 x_{2}+2 x_{3}+2 x_{4}+2 x_{5}+4 x_{6}+2 x_{7}-2 x_{8} \\
& 0=-4+5 x_{1}+5 x_{2}+5 x_{3}+5 x_{4}+5 x_{5}+6 x_{6}+6 x_{7}+10 x_{8} \\
& 0=2+4 x_{1}+4 x_{2}+4 x_{3}+4 x_{4}+4 x_{5}-5 x_{6}+3 x_{7}-7 x_{8}
\end{aligned}
$$

3.6 TABLE: 4 CUTS SLICING 908 EDGES OF THE 8-CUBE. 


\begin{tabular}{|c|c|}
\hline Cut 5.1 & $: 0=x_{1}+x_{2}+x_{3}+x_{4}+x_{5}$ \\
\hline Cut 5.2 & $: 0=-x_{1}+x_{2}+x_{3}+x_{4}+x_{5}$ \\
\hline Cut 5.3 & $: 0=3 x_{1}+x_{2}+x_{3}+x_{4}+x_{5}$ \\
\hline Cut 5.4 & $: \quad 0=-3 x_{1}+x_{2}+x_{3}+x_{4}+x_{5}$ \\
\hline Cut 6.1 & $x_{2}+x_{3}+x_{4}+x_{5}+x_{6}$ \\
\hline Cut 6.2 & $: 0=2 x_{1}+x_{2}+x_{3}+x_{4}+x_{5}+x_{6}$ \\
\hline Cut 6.3 & $: \quad 0=-2 x_{1}+x_{2}+x_{3}+x_{4}+x_{5}+x_{6}$ \\
\hline Cut 7.1 & $: 0=x_{1}+x_{2}+x_{3}+x_{4}+x_{5}+x_{6}+x_{7}$ \\
\hline Cut 7.2 & $: \quad 0=-x_{1}+x_{2}+x_{3}+x_{4}+x_{5}+x_{6}+x_{7}$ \\
\hline Cut 7.3 & $: 0=3 x_{1}+x_{2}+x_{3}+x_{4}+x_{5}+x_{6}+x_{7}$ \\
\hline Cut 7.4 & $: 0=-3 x_{1}+x_{2}+x_{3}+x_{4}+x_{5}+x_{6}+x_{7}$ \\
\hline Cut 8.1 & $x_{2}+x_{3}+x_{4}+x_{5}+x_{6}+x_{7}+x_{8}$ \\
\hline Cut 8.2 & $: 0=2 x_{1}+x_{2}+x_{3}+x_{4}+x_{5}+x_{6}+x_{7}+x_{8}$ \\
\hline Cut 8.3 & $: \quad 0=-2 x_{1}+x_{2}+x_{3}+x_{4}+x_{5}+x_{6}+x_{7}+x_{8}$ \\
\hline Cut 8.4 & $: 0=4 x_{1}+x_{2}+x_{3}+x_{4}+x_{5}+x_{6}+x_{7}+x_{8}$ \\
\hline Cut 8.5 & $: \quad 0=-4 x_{1}+x_{2}+x_{3}+x_{4}+x_{5}+x_{6}+x_{7}+x_{8}$ \\
\hline
\end{tabular}

3.7 TABLE: OTHER COMPUTER GENERATED CUTS.

3.8 Remark: Please notice a specialty in the proof to Theorem 3.2: For many lower bounds on $S(d, k-1)$ and $S(d, k)$ the corresponding sets of cuts are sort of incremental; to proceed from $S(d, k-1)$ to $S(d, k)$, one more cut from Table 3.7 is added. The reader might thus be inclined to believe: Any $k$ cuts which slice the maximum number $S(d, k)$ of edges

- can be reduced by one cut such that the remaining ones slice $S(d, k-1)$ edges;

- can be extended by a $(k+1)$ th cut to slice $S(d, k+1)$ edges.

Both conjectures however are wrong in general!

- No 2 of Paterson's optimal 5 cuts attain the maximum $S(6,2)=120$; they only reach 110 .

- And any 3 cuts of the 5-cube, each one optimal in the sense of slicing $S(5,1)=30$ edges, can together only reach a total of 66 edges rather than $70=S(5,3)$.

\section{Algorithms}

Our software was run concurrently on 16 Athlon ${ }^{\text {tm }} \mathrm{K} 6$ processors under Linux operating system. The values $S(5,2), S(5,3), S(5,4), S(6,3)$, and $S(7,2)$ in Table 3.3 have been obtained within about 2 months computation, a total of two and a half years CPU time!

A major property, invariance of the problem under cube symmetries was exploited to reduce the size of the search spaces: If a given subset $A$ of $\mathcal{E}_{d}$ can be sliced by $k$ cuts, then so can the subset $A^{\prime}$ obtained by arbitrarily permuting the $d$ coordinates: $\left(x_{1}, \ldots, x_{d}\right) \rightarrow\left(x_{\pi(1)}, \ldots, x_{\pi(d)}\right)$; similarly for some arbitrary reflection $\left(x_{1}, \ldots, x_{i}, \ldots, x_{d}\right) \rightarrow\left(x_{1}, \ldots,-x_{i}, \ldots, x_{d}\right)$. By this observation it suffices to consider only (sets of) cuts which are non-equivalent in the sense that they cannot be transformed into one each other by any combination of symmetries. 
After determining according to [15] all $2^{O\left(d^{2}\right)}$ cuts of the $d$-cube and the corresponding subsets of $\mathcal{E}_{d}$ they slice, we computed $k$-wise unions of these subsets. As explained above, one of the $k$ components may be restricted to non-equivalent cuts; the others however must each exhaust the full range. Being interested merely in the maximum cardinality of these unions, a branch-and-bound strategy came in effect.

To compute $S(5,3)$ and $S(5,4)$, we first applied dynamic programming techniques by determining all (symmetrically non-equivalent maximal) subsets of $\mathcal{E}_{5}$ which can be sliced by two cuts. As the 5-cube has 62 non-isomorphic and 47285 arbitrary cuts [16], this would expect a list of $62 \times 47285=2931670$ entries. Fortunately, many of them are either symmetrically equivalent or non-maximal or both: after removing these occurrences, only 150375 entries remained. This drastically reduced the search spaces for $S(5,3)$ and $S(5,4)$.

For $d>5$, such an approach was not possible any more, it simply exceeded our resources: Removing symmetrically equivalent or non-maximal entries on its own is a computationally expensive and memoryconsuming task which furthermore cannot efficiently be parallelized.

Thus for $S(6,3)$, we had no other choice than skim through all $566 \times 7.5 M \times 7.5 M=30 T$ combinations of cuts, each one represented by a subset of the $6 \cdot 2^{6-1}=192$ hypercube edges. Consequently this was the most time consuming task in our computations. Distributed on 16 machines, it took about 6 weeks of the total two months.

Starting with $S(6,4)$, the number of cases would become too large for exhaustive search. On the other hand, processing appropriate parts of the whole search space only, gives at least lower bounds. For this purpose it turned out a good heuristics to consider unions of large cuts, i.e., those which attain (or come close) to the largest possible cut of cardinality $S(d, 1)$. Note that this is only heuristics, see Remark 3.8.

\section{Conclusion}

This work refined the well-known cut number problem 'how many cuts are needed to slice all edges' to slicing numbers: 'how many edges can be sliced by a given number of cuts'. Computational methods [12] yielded new lower and upper bounds for small dimensions and number of cuts. These bounds were then extended to higher dimensions using combinatorial techniques (Lemma 3.1).

Although limited to low dimensions, we believe that the computational approach can reveal important insights and raise seminal conjectures on the behavior of the function $S(d, k)$ which, in turn, is tightly related to the cut number $C(d)$. Is it true, for example, that all values $S(d, k)$ are even? In other words: If an odd subset of $\mathcal{E}_{d}$ is sliced by $k$ hyperplanes, can one always add another edge to this set?

On the other hand, full power of computer aided research can only be exploited in connection with 'classical' theoretical considerations. Recall the cuts in Table 3.7 which in our opinion are very interesting for two reasons:

- Except for $S(6,4)$ and $S(8,4)$, all new lower bounds in Table 3.3 arise from these cuts. In other words: For most cases, they yield the largest known number of sliced edges.

- They generalize to arbitrary dimension $d$ and $k \leq d$, i.e., they form an entire class:

$$
H_{j}=\left\{x \in \mathbb{R}^{d}: 0=\sum_{i=1}^{d-1} x_{i}+j \cdot x_{d}\right\}, \quad j=\left\{\begin{aligned}
+1,-1,+3,-3, \ldots & : d \text { odd } \\
0,+2,-2,+4,-4, \ldots & : d \text { even }
\end{aligned}\right.
$$

We therefore believe these cuts deserve further analysis, and it was a computer that found them! 
Let us once more emphasize that nearly all lower bounds in Table 3.3 are attained by cuts either from (4) or (5). Among the only three exceptions are $S(6,5)$ and $S(6,4)$ which arise from the cuts of Paterson and in Table 3.4, respectively. Their 'singularity' seems to be related to the 'anomality' $C(5)=C(6)$ mentioned in Section 2; and it might indicate another anomality to happen at $C(7)=C(8)$ as the third exception $S(8,4)$ comes from the singular cut in Table 3.6.

The reader is invited to try own cuts for better lower bounds. Here again, a computer can give considerable support by releasing from the stupendous routine of determining and counting those edges which are sliced: Our web page [16] allows to interactively experiment with combinations of different cuts.

One of the authors ${ }^{\ddagger}$ wishes to fix a typographical error on his paper [3]. The last sentence of page 195 is not part of the omitted proof. The sentence, as a new paragraph, should be:

"However, it is easy to check that there are 4 copies of $H_{0}$ that can cover all the edges of a 3-face and all of its exterior edges in $c^{5}$."

\section{References}

[1] Egon Balas and Robert Jeroslow: "Canonical Cuts of the Unit Hypercube", pp.61-69 in SIAM J. Appl. Math. 23 No.1 (1972).

[2] M. Reza Emamy-Khansary: "On the cuts and cut number of the 4-cube", pp.211-227 in Journal of Combinatorial Theory Series A 41 (1986).

[3] M. Reza Emamy-Khansary: "On the covering cuts of $c^{d},(d \leq 5)$ ", pp.191-196 in Discrete Mathematics 68 (1988).

[4] M. Reza Emamy-Khansary: "On the Cut-number of the 5-Cube", pp.179-186 in Congressus Numerantium 72 (1990).

[5] M. Reza Emamy-Khansary, P. Pei, and C. Caiseda: "Cut-complexes and the greedy paths in the $n$ cube", pp.151-160 in Proceedings of the 8th Quadrenial International Conference on Graph Theory, Combinatorics, Algorithms, and Application Vol.I (1999), Y. Alavi et.al.

[6] Branko Grünbaum: "Polytopal Graphs", pp.201-204 in MAA Stud. Math. Vol.12, Math. Assoc. Amer., Washington (1975).

[7] A. Hajnal, W. Maass, P. Pudlák, M. Szegedy, G. Turán: "Threshold Circuits of Bounded Depth", pp.99-110 in Proc. 28th Ann. Symp. on Foundations of Computer Science (FOCS 1987).

[8] P. L. Hammer, B. Simone, Th. M. Liebling, and D. de Werras: "From Linear Separability to Unimodality: A Hierarchy of Pseudo-Boolean Functions", pp.174-184 in SIAM J. Disc. Math. 1 No.2 (1988).

[9] Victor Klee: "Shapes of the Future - Some Unresolved Problems in High-Dimensional Intuitive Geometry", in Proceedings of the 11th CCCG (1999), p.17

M. Reza Emamy-Khansary 
[10] Marvin L. Minsky and Seymour A. Papert: Perceptrons. MIT Press (1988).

[11] P. O’Neil: "Hyperplane cuts of an $n$-cube", pp.193-195 in Discrete Mathematics 1 (1971).

[12] J. Nievergelt etc al: "All the Needles in a Haystack: Can Exhaustive Search Overcome Combinatorial Chaos?”, pp. 254-274 in Computer Science Today, Springer LNCS 1000 (1995).

[13] M. Paterson, unpublished; see Example 3.72 in [14].

[14] Michael E. Saks: "Slicing the hypercube", pp.211-255 in Surveys in Combinatorics, Cambridge University Press (1993), Keith Walker (editor).

[15] Christian Sohler and Martin Ziegler: "Computing Cut Numbers", pp.73-79 in Proceedings of the 12th Annual Canadian Conference on Computational Geometry (CCCG 2000).

[16] Martin Ziegler: "Intermediate results for computing cut numbers", http://www.unipaderborn. de/cs/cubecuts 
